\title{
Evaluation of lobar lymph node metastasis in non-small cell lung carcinoma using modified total lesion glycolysis
}

\author{
Hitoshi Dejima, ${ }^{1,2}$, Hiroaki Kuroda ${ }^{1}$, Yuko Oya ${ }^{3}$, Noriaki Sakakura ${ }^{1}$, Yoshitaka Inaba ${ }^{4}$, Tsuneo Tamaki ${ }^{5}$, \\ Yasushi Yatabe $^{2}$, Yukinori Sakao ${ }^{1}$ \\ ${ }^{1}$ Department of Thoracic Surgery, ${ }^{2}$ Department of Pathology and Molecular Diagnostics, ${ }^{3}$ Department of Thoracic Oncology, ${ }^{4}$ Department of \\ Diagnostic and Interventional Radiology, Aichi Cancer Center Hospital, Nagoya, Japan; ${ }^{5}$ Nagoya Radiological Diagnosis Foundation, Nagoya, Japan \\ Contributions: (I) Conception and design: H Dejima, H Kuroda; (II) Administrative support: Y Sakao; (III) Provision of study materials or patients: \\ N Sakakura, Y Inaba, T Tamaki; (IV) Collection and assembly of data: H Dejima, N Sakakura, Y Inaba, T Tamaki, Y Yatabe; (V) Data analysis and \\ interpretation: H Dejima, Y Oya; (VI) Manuscript writing: All authors; (VII) Final approval of manuscript: All authors. \\ Correspondence to: Yukinori Sakao, MD, PhD. 1-1 Kanokoden, Chikusa-ku, Nagoya, Aichi, 464-8681, Japan. Email: ysakao@aichi-cc.jp.
}

Background: Volumetric parameters based on 3-dimensional reconstruction have recently been introduced for cancer staging. We aimed to improve the ability to diagnose hilar lymph node metastasis in patients with non-small cell lung cancer.

Methods: We evaluated 142 patients with non-small cell lung cancer who underwent right upper lobectomy and radical lymph node dissection. Metastatic involvement of right upper lobar lymph nodes was assessed using high-resolution computed tomography (HRCT) and 18F-2-floro-2-deoxyglucose-positron emission tomography/computed tomography (FDG-PET/CT).

Results: On receiver operating characteristic (ROC) curve analysis, the area under the curves (AUC) for short axis, maximum of standardized uptake value $\left(\mathrm{SUV}_{\text {max }}\right)$, total lesion glycolysis (TLG) and modified TLG (mTLG) were $0.79,0.77,0.76$, and 0.87 , respectively. The sensitivity, specificity, positive predictive value (PPV), and negative predictive value (NPV) of mTLG, using the optimal cut off value (2.45), for diagnosis of lobar lymph node metastasis were $71 \%, 88 \%$, 44\%, and 96\%, respectively. Hilar asymmetric uptake (HAU) of FDG was larger in true-positive cases than in false-negative cases $(\mathrm{P}<0.01)$. Furthermore, the size of metastatic foci in the lymph node was smaller in false-negative cases $(\mathrm{P}=0.012)$.

Conclusions: Modified TLG is a good parameter to diagnose metastatic right upper lobar lymph nodes. Micrometastasis in the lymph node is difficult to predict using the current diagnostic method. However, more careful evaluation is required in patients with symmetric FDG accumulation at hilar region because hilar lymph nodes respond to various causes such as benign pulmonary diseases.

Keywords: Total lesion glycolysis (TLG); hilar lymph node; positron emission tomography/computed tomography (PET/CT); lymph node metastasis; sublobar resection

Submitted Feb 06, 2018. Accepted for publication Oct 17, 2018.

doi: $10.21037 /$ jtd.2018.11.40

View this article at: http://dx.doi.org/10.21037/jtd.2018.11.40

\section{Introduction}

Clinical diagnosis of lymph node metastasis is a critical input for formulating the treatment strategy for primary lung cancer. Metastatic involvement of mediastinal lymph node is one of the key considerations during evaluation of the indications for surgery. On the other hand, hilar lymph nodes are typically neglected during assessment of surgical indication, regardless of their metastatic involvement. However, the perihilar lymph nodes are surrounded by pulmonary artery, pulmonary vein and bronchus, and its anatomical features require careful surgical procedure. In addition, dissection of hilar lymph nodes with metastasis often requires the use of advanced techniques and invasive 
procedures such as pulmonary angioplasty, bronchoplasty, bilobectomy, and pneumonectomy. Furthermore, it is difficult to dissect the upper lobar lymph nodes in right middle or lower lobectomy without upper lobectomy. For these reasons, accurate preoperative detection of metastatic involvement of perihilar lymph nodes confers a distinct advantage to the operating surgeon in terms of safety as well as surgical outcomes.

Swelling of hilar lymph nodes is not specific to carcinoma but may also occur in benign lung disease such as infection, emphysema, silicosis, sarcoidosis, cardiac disease, and nonspecific inflammation. Therefore, the diagnostic value of swollen hilar lymph nodes has been widely contested. For instance, Glazer and colleagues (1985) stated that "Accurate staging of the pulmonary hilum in patients with lung cancer is not currently possible using MRI, CT, or oblique tomography" (1). Positron emission tomographycomputed tomography (PET-CT), which employs both CT (for morphological evaluation) and PET (for evaluation of tumor activity), has been used to assess metastatic involvement of hilar lymph nodes. Studies have shown that PET-CT is useful for evaluation of mediastinal lymph nodes; however, its' efficacy for assessment of hilar lymph node involvement is not well-established $(2,3)$. In addition to PET-CT, use of volume-based parameters measured by 3-dimensional (3D) reconstruction has recently attracted much interest.

Total lesion glycolysis (TLG) is a semi-quantitative parameter of metabolic activity of tumors. TLG is calculated by multiplication of the mean standardized uptake value $\left(\mathrm{SUV}_{\text {mean }}\right)$ with the metabolic tumor volume (MTV). TLG is a quantitative parameter obtained from MTV and 18F-2-floro-2-deoxyglucose (FDG) accumulation intensity; it has been suggested to better reflect global metabolic activity in whole tumors. TLG has been used as a quantitative parameter of tumor activity in the context of various cancers $(4,5)$. In this study, we investigated the accuracy of clinical diagnosis of hilar lymph node metastasis by using parameters that combined CT and PET.

\section{Methods}

\section{Patients}

A total of 289 patients underwent right upper lobectomy and radical lymph node dissection, as a complete resection, at the Aichi Cancer Center, Nagoya, Japan in the period from April 2012 to September 2016. Of these, only patients with primary non-small cell lung carcinoma were considered for inclusion in this study. Patients who received any induction therapy were excluded. We also excluded patients for whom complete clinical data or image analysis (due to mismatch with analysis software) was not available. Written informed consent was obtained from all patients. This study was approved by the Aichi Cancer Center Hospital Ethical Committee. Our study was performed in full accordance with local Institutional Review Board (IRB) guidelines (No. 2017-1-114). Clinical data was obtained from the hospital records. All radiological images were independently evaluated by two radiologists (Tsuneo Tamaki and Yoshitaka Inaba) who were blinded to clinical data. In the same way, all surgical specimens were evaluated by a pathologist (Yasushi Yatabe).

\section{High-resolution computed tomography (HRCT) image analysis}

We targeted the right upper lobar lymph node for this study. Lobar lymph nodes were defined as "\#12" according to World Health Organization (WHO) Classification (6). Further, we defined right upper lobar lymph node as "\#12u" (Figure 1A). CT scan was performed using 64 or 80-detector-row CT scanner (Aquilion PRIME; Toshiba Medical Systems Co., Japan). Whole chest was scanned in 90 seconds after injection of $1,224.8 \mathrm{mg} / 2 \mathrm{~mL} / \mathrm{kg}$ of Iopamidol during a breath-hold at deep inspiration phase in supine position; 1-mm thick high-resolution images were reconstructed using standard spatial-frequency reconstruction algorithm. Data was transferred to a commercially available workstation in DICOM format (Synapse Vincent; Fujifilm Medical Co., Japan). 3D images were reconstructed using the work station, and the volumes of primary tumors and \#12u lymph nodes were calculated (Figure 1B).

\section{PET-CT image analysis}

PET-CT images were acquired according to our standard imaging protocol using dedicated PET/CT scanner (Biograph 40; SIEMENS Healthcare Japan Co., Japan). Patients fasted for at least 6 hours prior to injection of $3.8 \mathrm{MBq} / \mathrm{kg}$ of FDG. Subsequently, patients were scanned 90 minutes after injection of FDG. Summary of our protocol is described in our earlier paper (7). $\mathrm{SUV}_{\text {max }}, \mathrm{SUV}_{\text {mean }}$, and MTV of tumors and lymph nodes were calculated using a SIEMENS MMWP workstation (Figure 1C). 

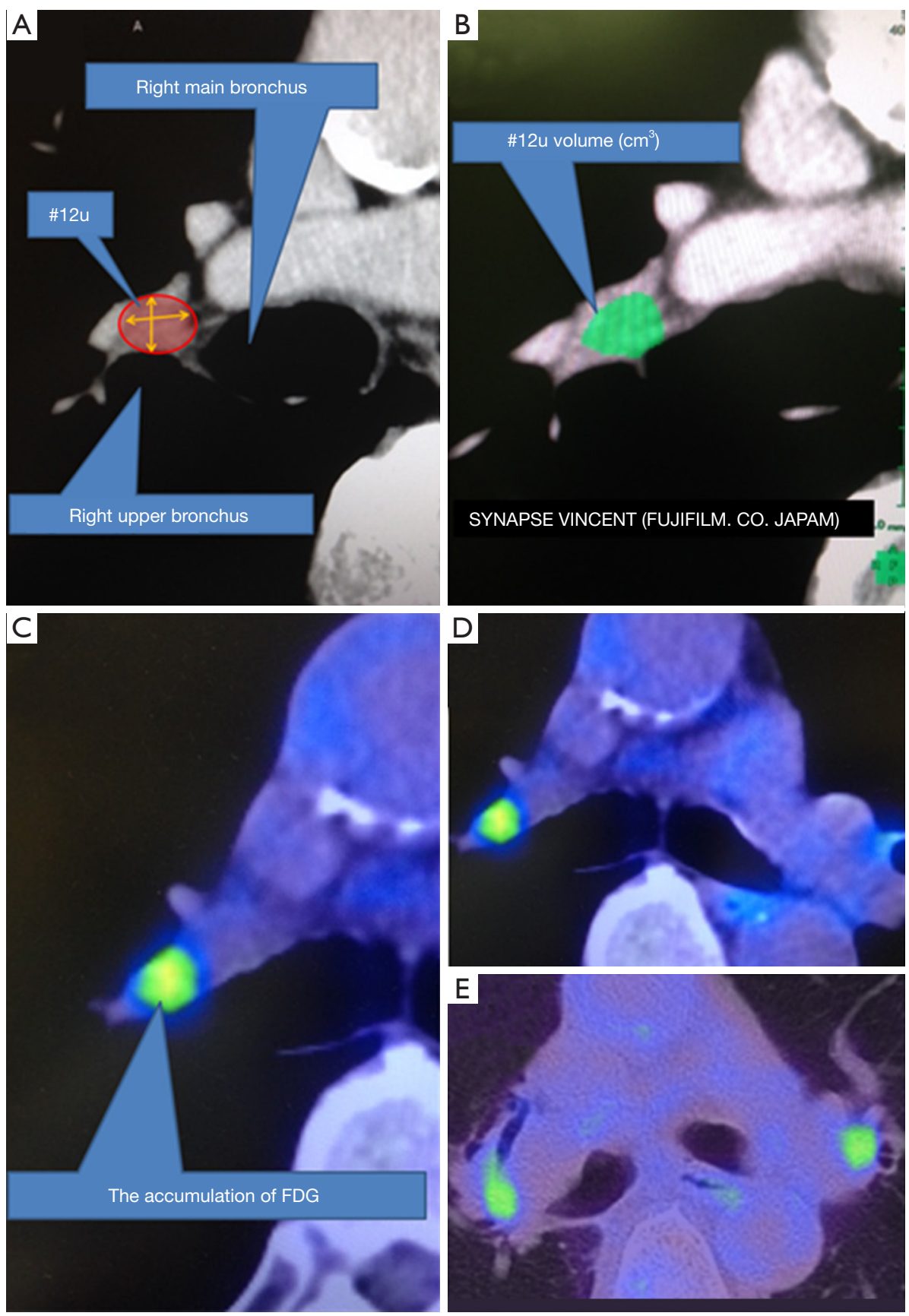

Figure 1 Radiological image analysis. (A) \#12u was defined as adjacent to the right upper lobar bronchus. It is surrounded by right main bronchus, right upper lobar bronchus, A2b of pulmonary artery branch, and right main pulmonary artery; (B) volume of \#12u lymph node was measured by Synapse Vincent software; (C) SUV was measured by PET-CT. It was used for calculation of each parameter; (D) a typical image of asymmetric uptake and (E) symmetric uptake. FDG, ${ }^{18} \mathrm{~F}$-2-floro-2-deoxyglucoce; SUV, standardized uptake value; PET-CT, positron emission tomography/computed tomography. 


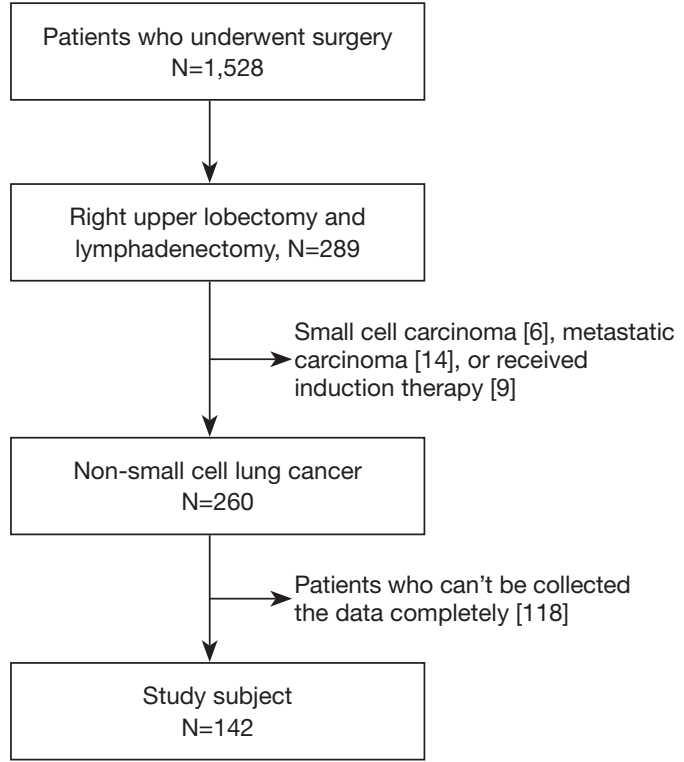

Figure 2 A patient selection chat shows 142 patients who underwent right upper lobectomy and radical lymph node dissection for nonsmall cell lung carcinoma were selected as our study cohort.

Table 1 Patient characteristics

\begin{tabular}{lc}
\hline Parameter & All $(\mathrm{n}=142)$ \\
\hline Age (years, median) & $68[41-87]$ \\
Sex & \\
Male & 87 \\
Female & 55 \\
BI (median) & $445[0-3,000]$ \\
CEA (ng/mL, median) & $3.3(0.5-559)$ \\
Tumor appearance (CT scan) & \\
Pure GGN & 2 \\
Part solid GGN & 51 \\
Solid & 89 \\
Histological type & \\
AD & 107 \\
SQ & 20 \\
Others & 15 \\
\#12u (pathological) & \\
Normal & 17 \\
Metastasis & \\
\hline
\end{tabular}

Data are presented as $\mathrm{n}$ or median (range). Bl, Brinkman index; CEA, carcinoembryonic antigen; CT, computed tomography; GGN, ground-glass nodules; AD, adenocarcinoma; $S Q$, squamous cell carcinoma.
One technical assistant who was blinded to the clinical data independently calculated these parameters. TLG and modified TLG of a lesion were calculated as $\mathrm{SUV}_{\text {mean }} \times \mathrm{MTV}$ and $\mathrm{SUV}_{\text {mean }} \times$ volume measured by CT, respectively. The difference of FDG uptake between both sides was evaluated by two radiologists with eyeball approach (Figure 1D,E).

\section{Statistical analysis}

All statistical analyses were performed using JMP 12 software (SAS Institute, Cary, North Carolina, USA). Between-group differences were assessed with MannWhitney U-test, Chi-squared test, Student's $t$-test, and logistic regression. Receiver operating characteristic (ROC) curve analyses were performed for variables that were associated with $\mathrm{P}$ values $<0.05$ on univariate analysis using the logistic regression test or the Cox proportional hazards model. All $\mathrm{P}$ values are two-sided, and $\mathrm{P}$ values of $<0.05$ were considered statistically significant.

\section{Results}

\section{Patient characteristics}

In total, 142 patients were enrolled in this study. They underwent right upper lobectomy and radical lymph node dissection for non-small cell lung carcinoma, and their clinical data was evaluated. Main reason why 118 patients can't be completely collected the data was that theses patient had PET examination in another institution (Figure 2). Seventeen patients were found to have metastatic involvement of \#12u lymph node based on histopathological examination after operation (Table 1). Incidentally, no patient was diagnosed with hilar lymph node metastasis with histopathology, such as endobronchial ultrasoundguided transbronchial needle aspiration (EBUS-TBNA) and mediastinoscopy, before operation.

The cases of ground-glass nodules (GGN) and small nodules of diameter $\leq 10 \mathrm{~mm}$ were in the \#12u non-metastatic group. T-CD and T-SUVmax were significantly higher in the $\# 12 \mathrm{u}$ metastasis group as a primary tumor factor $(\mathrm{P}=0.04$ and $<0.01$, respectively). In addition, pathological $\mathrm{N} 2$ metastasis, \#12u-short axis, \#12u-volume, \#12u-SUV $\mathrm{max}_{\max }$, \#12u-TLG, and \#12u-mTLG were significantly higher in the $\# 12 \mathrm{u}$ metastasis group as a lymph node factor $(\mathrm{P}<0.01$ with each factors). Skip N2 metastasis (N1negative and N2-positive) were 3 out of 125 cases (Table 2). 
Table 2 Comparison between the groups with and without \#12u metastasis

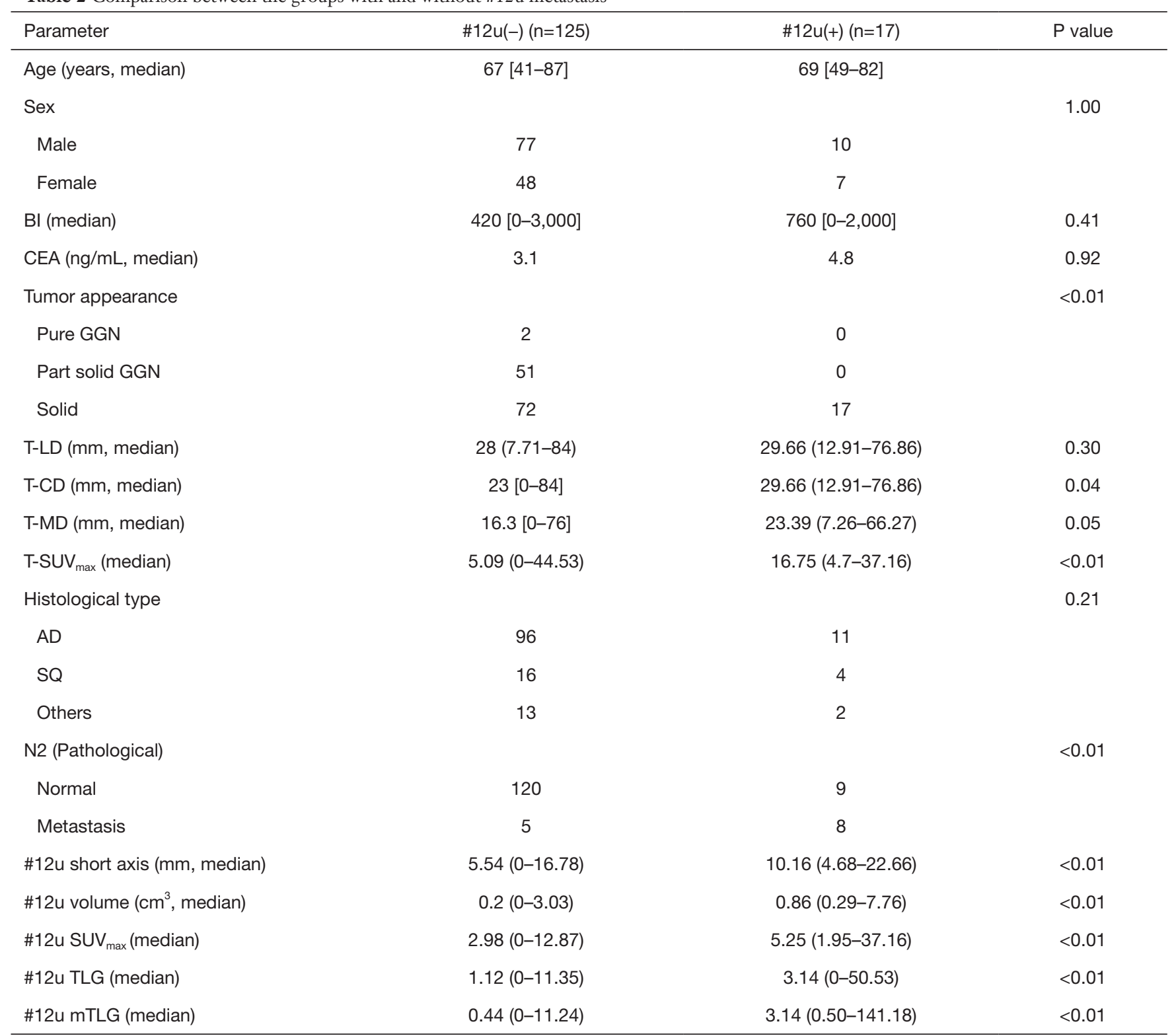

Data are presented as $\mathrm{n}$ or median (range). BI, Brinkman index; CEA, carcinoembryonic antigen; GGN, ground-glass nodule; T-LD, wholetumor dimension in the lung window setting; T-CD, tumor consolidation dimension; T-MD, tumor dimension in the mediastinal window

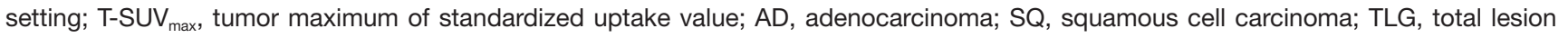
glycolysis; mTLG, modified TLG.

None of the patients in this cohort had diabetes.

\section{ROC analyses of parameters based on $2 D$ and $3 D$ imaging findings of \#12u lymph nodes}

Table 3 shows the result of ROC analyses of 2D and 3D image-based parameters. The area under the curves
(AUC) of short axis and SUVmax based on 2D image were 0.79 and 0.77 , respectively. These are commonly used parameters for assessment of lymph node involvement. Volume, TLG, and mTLG calculated from 3D images were superior to 2D-based parameters. According to AUC, mTLG was found to be the best parameter in this study (Figure 3). AUC, sensitivity, specificity, positive predictive 
Table 3 ROC analysis of various parameters for \#12u metastasis

\begin{tabular}{llccccc}
\hline Parameter & AUC & Cut off & Sensitivity (\%) & Specificity (\%) & PPV (\%) & NPV (\%) \\
\hline Short axis & 079 & 10.16 & 53 & 29 & 50 & 94 \\
Volume & 0.85 & 0.66 & 76 & 70 & 34 & 80 \\
SUV $_{\max }$ & 0.77 & 3.80 & 86 & 70 & 96 \\
TLG & 0.76 & 2.48 & 71 & 88 & 98 \\
mTLG & 0.87 & 2.45 & 98 & 44 & 96 \\
\hline
\end{tabular}

ROC, receiver operating characteristic; AUC, area under the curves; PPV, positive predictive value; NPV, negative predictive value; $S U V_{\text {max }}$ maximum of standardized uptake value; TLG, total lesion glycolysis; mTLG, modified TLG.

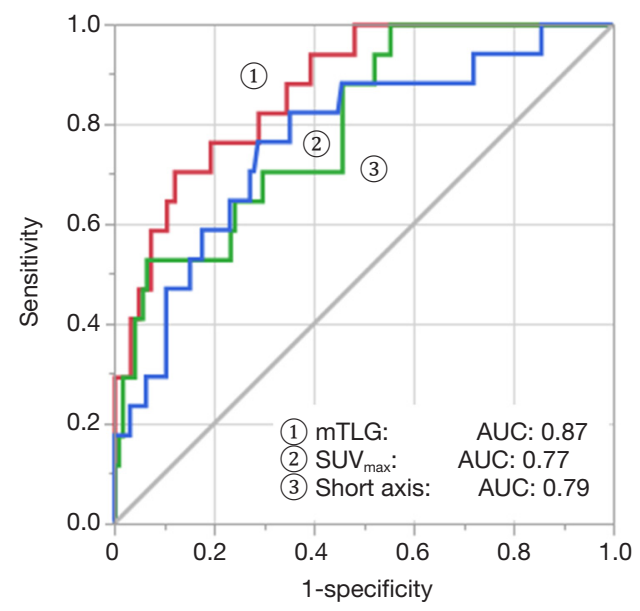

Figure 3 Results of ROC curve analysis showing diagnostic performance of mTLG in comparison to that of currently used parameters such as $\mathrm{SUV}_{\max }$ and short axis. ROC, receiver operating characteristic; mTLG, modified total lesion glycolysis; $\mathrm{SUV}_{\max }$, maximum standardized uptake value.

value (PPV), and negative predictive value (NPV) for $\# 12 \mathrm{u}$ lymph node metastasis was $0.87,71 \%, 88 \%, 44 \%$, and $96 \%$, respectively. The optimal cutoff point of mTLG for \#12u lymph node metastasis was 2.45 .

\section{Analysis of false-positive and false negative cases}

We examined the relationship between true-positive and false-positive cases using the cutoff value of 2.45 calculated from the ROC curve. No significant differences were observed between false-positive and true-positive groups with respect to Brinkman index (BI), neutrophil/lymphocyte ratio (N/L), carcinoembryonic antigen (CEA), dimension of primary tumor on CT with lung and mediastinum setting, and $\mathrm{SUV}_{\max }$ and TLG of primary tumor. Only the number
Table 4 Comparison between FP and TP groups

\begin{tabular}{lccc}
\hline Parameter & FP $(\mathrm{n}=15)$ & $\mathrm{TP}(\mathrm{n}=12)$ & P value \\
\hline BI (med) & 720.00 & 850.00 & 0.23 \\
$\mathrm{~N} / \mathrm{L}(\mathrm{med})$ & 3.39 & 2.97 & 0.44 \\
CEA (ng/mL, med) & 6.40 & 3.90 & 0.58 \\
T-CD (mm, med) & 39.00 & 32.80 & 0.60 \\
T-MD (mm, med) & 30.00 & 27.50 & 0.46 \\
T-SUV $_{\text {max }}(\mathrm{med})$ & 17.14 & 17.04 & 0.25 \\
T-TLG (med) & 76.87 & 41.47 & 0.47 \\
HAU (n) & 4.00 & 10.00 & $<0.01$ \\
\hline
\end{tabular}

FP, false-positive; TP, true-positive; BI, Brinkman index; N/L, neutrophil/lymphocyte ratio, CEA, carcinoembryonic antigen; $\mathrm{T}-\mathrm{CD}$, tumor consolidation dimension; T-MD, tumor dimension in the mediastinal window setting; T-SUV $\mathrm{max}_{\max }$, tumor maximum of standardized uptake value; T-TLG, tumor total lesion glycolysis; $\mathrm{HAU}$, hilar asymmetric uptake.

of patients who showed hilar asymmetric uptake (HAU) on PET was significantly higher in the TP group (Table 4).

Furthermore, we examined the lymph nodes showing false negative microscopically. The number of false-negative cases was five. Each total cross-sectional area of metastatic foci in the lymph node was $0.01,4,4.6,10$, and $24 \mathrm{~mm}^{2}$, respectively. The number of these cases was significantly smaller than that of true positive cases $(\mathrm{P}=0.012)$ (Figure 4).

\section{ROC analysis of mTLG separated the cases with bilar uptake pattern on PET}

Forty-six patients had HAU and 96 patients had hilar symmetric uptake on PET. Table 5 showed that the volume calculated using CT scan and mTLG of \#12u was a good parameter for making the diagnosis of metastasis. AUC, sensitivity, specificity, PPV, and NPV using volume and 
mTLG were 0.88 and $0.89,78 \%$ and $79 \%, 88 \%$ and $88 \%, 73 \%$ and $73 \%$, and $90 \%$ and $91 \%$, respectively. On the other hand, patients who had hilar symmetric uptake showed that AUC using various parameters such as short axis, volume, SUVmax, TLG, and mTLG of \#12u were $0.71,0.76,0.53,0.58$, and 0.77 , respectively.

\section{Discussion}

The utility of CT and PET for the detection of metastatic involvement of mediastinal lymph nodes is wellacknowledged; however, their relevance in the context of hilar lymph node involvement is not clear $(2,3)$. We succeeded in improving the accuracy of detection of hilar lymph node involvement with use of volume-based parameters (such as, TLG) determined with combined use of CT and PET. However, MTV for calculation of TLG

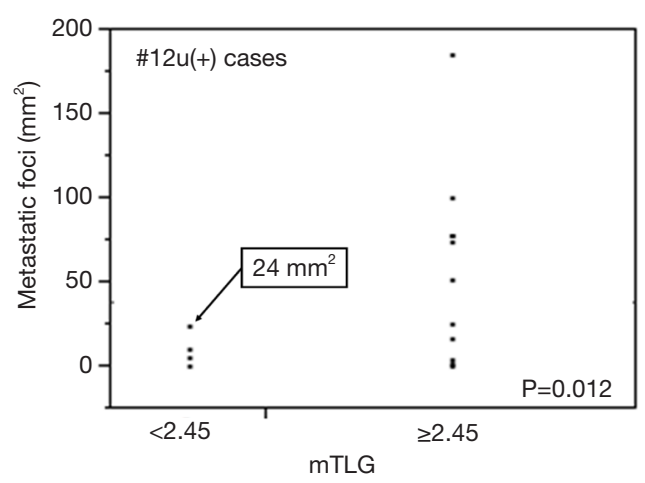

Figure 4 Total cross-sectional area of metastatic foci was calculated microscopically in all \#12u(+) cases. The mTLG $<2.45$ cases were significantly lesser the cross-sectional area of metastatic foci than $\geq 2.45$ cases. And all mTLG $<2.45$ cases were less than $25 \mathrm{~mm}^{2}$ in size. mTLG, modified total lesion glycolysis. is greatly affected by partial volume effect; therefore, we must be careful to avoid measurement errors attributable to the latter (8). Kim and colleagues reported a cut off value of SUV for measurement of MTV and expressed it as $\mathrm{TLG}_{2.5}$ or $\mathrm{TLG}_{3.0}$ (4). To minimize measurement errors attributable to partial volume effect, we measured the volume of lymph nodes using HRCT, and determined SUV of lymph node from PET; in other words, volume and SUV were calculated separately in this study. Then, we defined it as mTLG. As a result, the mTLG was associated with the largest AUC when compared with those of TLG and other currently used parameters (Table 2, Figure 3). In the entire cohort, however, false-positive rate of all parameters including $\mathrm{mTLG}$ was $\geq 50 \%$, which is not a clinically negligible value. According to our results, falsepositive cases exhibited more symmetric accumulation at the hilar lymph nodes, which was significantly different from that observed in the true-positive group. FDG not only accumulates in the tumor but also in macrophages and reactive fibroblasts in the lymph nodes (9). For that reason, hilar lymph nodes respond to various conditions such as pulmonary emphysema, tuberculosis, non-specific inflammation, silicosis, sarcoidosis, smoking, and air pollution, which may affect the accuracy of staging of lung cancer (3,10-13). In a study by Ose and colleagues, FP findings were more frequently encountered in patients with elevated white blood cell counts and in smokers (14). In this study, significant differences were observed between false-positive and true-positive cases with respect to HAU. HAU is typically associated with tumors because most of benign diseases including tuberculosis are characterized by bilaterally symmetric accumulation $(3,10)$. As shown in Table 5, we could distinguish the accumulation of \#12u as symmetry or asymmetry using PET, and mTLG and volume were useful for detecting metastasis. According to

Table 5 ROC analysis of various parameters for \#12u metastasis among patients having asymmetric uptake on the hilar lymph node on PET-CT

\begin{tabular}{|c|c|c|c|c|c|c|}
\hline Parameter & AUC & Cut off & Sensitivity (\%) & Specificity (\%) & PPV (\%) & NPV (\%) \\
\hline Short axis & 0.81 & 10.3 & 64 & 91 & 75 & 85 \\
\hline Volume & 0.88 & 0.77 & 78 & 88 & 73 & 90 \\
\hline $\mathrm{SUV}_{\max }$ & 0.70 & 5.25 & 57 & 76 & 50 & 80 \\
\hline TLG & 0.65 & 15.9 & 35 & 100 & 100 & 78 \\
\hline mTLG & 0.89 & 2.45 & 79 & 88 & 73 & 91 \\
\hline
\end{tabular}

ROC, receiver operating characteristic; PET-CT, positron emission tomography/computed tomography; AUC, area under the curves; PPV, positive predictive value; NPV, negative predictive value; $\mathrm{SUV}_{\max }$, maximum of standardized uptake value; TLG, total lesion glycolysis; mTLG, modified TLG. 
the result of this study, in cases with asymmetric uptake, the cutoff value of mTLG and volume for detection of metastasis for $\# 12 \mathrm{u}$ were 2.45 and $0.77 \mathrm{~cm}^{3}$, respectively. An imagination of this indication is a sphere with roughly $11 \mathrm{~mm}$ in diameter and 3 as $\mathrm{SUV}_{\text {mean }}$ accumulation. The conventional parameter, short axis, was limited both sensitivity and specificity compare with mTLG. However, it performed pretty much equally when we would select HAU cases (Sensitivity, specificity, PPV, and NPV of short axis and mTLG limited to the HAU cases were 64\%, 91\%, $75 \%$ and $85 \% ; 79 \%, 88 \%, 73 \%$ and $91 \%$, respectively.) In cases with symmetric uptake, no parameter was useful for detection. Currently, we cannot distinguish cancer metastasis from systemic inflammation using radiological images. On the other hand, none of the patients in the $\# 12 \mathrm{u}$ metastatic group exhibited mTLG value of zero (i.e., hilar lymph nodes not identifiable on HRCT or no accumulation on PET), or had GGN with $<10 \mathrm{~mm}$ diameter of the solid part on HRCT. This result is consistent with the current knowledge base and with the results of our previous research $(15,16)$.

In the false negative cases, we examined the metastatic lymph node microscopically. All FN cases showed the total cross-sectional area of metastatic foci was less than $25 \mathrm{~mm}^{2}$ in the lymph node. Similarly, it has been reported that metastatic foci smaller than $5 \mathrm{~mm}$ can be hard to detect by PET-CT (2). We should recognize that detection of micrometastasis in the lymph node using radiological imaging is currently unavailable. The new method for detecting micrometastasis in lymph nodes should be devised.

As a future prospect, we believe that our findings can be applied to determine the indication for limited resection and aid surgical decision-making for primary lesions in other lung lobes. In recent years, limited resection is increasingly employed for patients with comorbid conditions, elderly patients, those with poor respiratory function or in patients with early-stage non-small cell lung cancer (17-19). Limited resection, especially wedge resection, is a less invasive alternative to lobectomy. However, limited resection does not permit adequate lymph node dissection, which is a limitation (20). Indeed, evaluation of the need for lymph node dissection based on mTLG may help identify candidates for limited resection. Furthermore, we often experience early locoregional recurrence around the $\# 12 \mathrm{u}$ after operation for primary lung cancer in the right lower lobe. Yamauchi and colleagues reported a dynamic pattern of post-curative resection recurrence in patients with non-small cell lung cancer, which was characterized by double-peak of hazard rate. There was an early peak at approximately 10 months (21). The undetectable micrometastatic foci may exist in different biologically steady states during tumor dormancy. It does not usually promote tumor growth. However, micrometastasis may transiently accelerate metastatic growth following surgical excision of the primary tumor. The transition from dormancy to active state may be triggered by surgical stimulus $(22,23)$. Patients with suspected \#12u metastasis from lung cancer in the right lower lobe may be considered candidates for additional treatment such as \#12u lymph node dissection, adjuvant chemotherapy or perioperative radiotherapy. The results of our study may provide a valuable basis for the development of such future lung cancer therapeutics.

Our study has some limitations. First, because this cohort was enrolled from patients who had undergone surgery, it comprised mainly of patients with early-stage lung cancer. Further, histopathological examination to determine contralateral hilar lymph node involvement was not performed in any of the patients. The influence of patient-selection bias on our results cannot be ruled out. Furthermore, a relatively small number of patients had confirmed metastatic involvement of hilar lymph nodes. Next, during radiological evaluation of symmetric versus asymmetric hilar uptake on PET, only the color depth of hotspot at bilateral hilar region was taken into consideration, while the contralateral hilar SUV was not calculated. This might have introduced an element of information bias. Lung cancer staging based on imaging is typically challenging because appearance of lymph nodes tends to differ depending on each station such as right hilar, left hilar, upper mediastinum, and lower mediastinum. Therefore, we investigated only the \#12u lymph nodes to improve reproducibility.

\section{Conclusions}

In conclusion, it is difficult to detect metastatic lymph nodes, especially at the $\# 12 \mathrm{u}$ station. However, combined use of CT and PET may offer a distinct leverage in this respect and improve the accuracy of preoperative staging. Especially, HAU on PET is important. It is useful not only mTLG but also short axis when we select the HAU cases. Whereas, all parameters couldn't improve the diagnostic performance in the cases with symmetric uptake. Technological advances have improved the accuracy of 
imaging diagnosis over the years.

\section{Acknowledgements}

We thank Mr. Tanaka $\mathrm{H}$ for his excellent technical assistance, and all members of Nagoya Radiological Diagnosis Foundation, Nagoya, Japan for their examination and analysis of PET-CT.

\section{Footnote}

Conflicts of Interest: The authors have no conflicts of interest to declare.

Ethical Statement: This study was approved by the Aichi Cancer Center Hospital Ethical Committee. Our study was performed in full accordance with local IRB guidelines (No. 2017-1-114). Each patient was informed that his clinical data could be used for various clinical studies, and written informed consent for this report was obtained on this basis.

\section{References}

1. Glazer GM, Gross BH, Aisen AM, et al. Imaging of the pulmonary hilum: A prospective comparative study in patients with lung cancer. AJR Am J Roentgenol 1985;145:245-8.

2. Nomori H, Watanabe K, Ohtsuka T, et al. The size of metastatic foci and lymph nodes yielding false-negative and false-positive lymph node staging with positron emission tomography in patients with lung cancer. J Thorac Cardiovasc Surg 2004;127:1087-92.

3. Pak K, Park S, Cheon GJ, et al. Update on nodal staging in non-small cell lung cancer with integrated positron emission tomography/computed tomography: a metaanalysis. Ann Nucl Med 2015;29:409-19.

4. Kim BH, Kim SJ, Kim K, et al. High metabolic tumor volume and total lesion glycolysis are associated with lateral lymph node metastasis in patients with incidentally detected thyroid carcinoma. Ann Nucl Med 2015;29:721-9.

5. Van de Wiele C, Kruse V, Smeets P, et al. Predictive and prognostic value of metabolic tumour volume and total lesion glycolysis in solid tumours. Eur J Nucl Med Mol Imaging 2013;40:290-301.

6. Travis WD, Brambilla E, Burke AP, et al. WHO Classification of Tumours of the Lung, Pleura, Thymus and Heart. Fourth edition. WHO Press, 2015.
7. Yoshida T, Tanaka H, Kuroda H, et al. Standard uptake value on $18 \mathrm{~F}-\mathrm{FDG}-\mathrm{PET} / \mathrm{CT}$ is a predictor of EGFR T790M mutation status in patients with acquired resistance to EGFR-TKIs. Lung Cancer 2016;100:14-9.

8. Soret M, Bacharach SL, Buvar I. Partial-volume effect in PET tumor imaging. J Nucl Med 2007;48: 932-45.

9. Kubota R, Yamada S, Kubota K, et al. Intratumoral Distribution of fluorine- 18-FluorodeoxyglucoseIn Vivo: High Accumulation in Macrophages and Granulation Tissues Studied by Microautoradiography. J Nucl Med 1992;33:1972-80.

10. Liao CY, Chen JH, Liang JA, et al. Meta-analysis study of lymph node staging by $18 \mathrm{~F}-\mathrm{FDG}$ PET/CT scan in nonsmall cell lung cancer: Comparison of TB and non-TB endemic regions. Eur J Radiol 2012;81:3518-23.

11. An YS, Sun JS, Park KJ, et al. Diagnostic performance of (18)F-FDG PET/CT for lymph node staging in patients with operable non-small-cell lung cancer and inflammatory lung disease. Lung 2008;186:327-36.

12. Shreve PD, Anzai Y, Wahl RL. Pitfalls in oncologic diagnosis with FDG PET imaging: physiologic and benign variants. Radiographics 1999;19:61-77.

13. Takamochi K, Yoshida J, Murakami K, et al. Pitfalls in lymph node staging with positron emission tomography in nonsmall cell lung cancer patients. Lung Cancer 2005;47:235-42.

14. Ose N, Sawabata N, Minami M, et al. Lymph node metastasis diagnosis using positron emission tomography with 2-[18F] fluoro-2-deoxy-D-glucose as a tracer and computed tomography in surgical cases of non-small cell lung cancer. Eur J Cardiothorac Surg 2012;42:89-92.

15. Sakao Y, Kuroda H, Mun M, et al. Prognostic Significance of Tumor Size of Small Lung Adenocarcinomas Evaluated with Mediastinal Window Settings on Computed Tomography. PLoS one 2014;9:e110305.

16. Sakakura N, Inaba $Y$, Yatabe $Y$, et al. Estimation of the pathological invasive size of pulmonary adenocarcinoma using high-resolution computed tomography of the chest: A consideration based on lung and mediastinal window settings. Lung Cancer 2016;95:51-6.

17. Okami J, Higashiyama $M$, Asamura H, et al. Pulmonary Resection in Patients Aged 80 Years or Over with Clinical Stage I Non-small Cell Lung Cancer. J Thorac Oncol 2009;4:1247-53.

18. Aokage K, Yoshida J, Hishida T, et al. Limited resection for early-stage non-small cell lung cancer as functionpreserving radical surgery: a review. Jpn J Clin Oncol 2017;47:7-11. 
19. Koike T, Koike T, Sato S, et al. Lobectomy and limited resection in small-sized peripheral non-small cell lung cancer. J Thorac Dis 2016;8:3265-74.

20. Tsutani Y, Miyata Y, Nakayama H, et al. Appropriate sublobar resection choice for ground glass opacitydominant clinical stage IA lung adenocarcinoma: wedge resection or segmentectomy. Chest 2014;145:66-71.

21. Yamauchi Y, Thomas M, Seyer S, et al. The dynamic pattern of recurrence in curatively resected non-small cell lung cancer patients: Experiences at a single institution.

Cite this article as: Dejima H, Kuroda H, Oya Y, Sakakura N, Inaba Y, Tamaki T, Yatabe Y, Sakao Y. Evaluation of lobar lymph node metastasis in non-small cell lung carcinoma using modified total lesion glycolysis. J Thorac Dis 2018;10(12):69326941. doi: $10.21037 / j t d .2018 .11 .40$
Lung Cancer 2015;90:224-9.

22. Demicheli R, Fornili M, Ambrogi F, et al. Recurrence dynamics for non-small-cell lung cancer: effect of surgery on the development of metastases. J Thorac Oncol 2012;7:723-30.

23. Zhu JF, Feng XY, Zhang XW, et al. Time-varying pattern of postoperative recurrence risk of early-stage (T1aT2bN0M0) non-small cell lung cancer (NSCLC): results of a single-center study of 994 Chinese patients. PLoS One 2014;9:e106668. 